\title{
El cambio estratégico en la adquisición del conocimiento: la metodología microgenética
}

\author{
Mercé Garcia-Mila, Sandra Gilabert y Nubia Rojo \\ Universidad de Barcelona
}

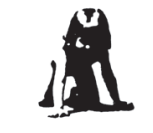

\section{Resumen}

El desarrollo de estrategias cognitivas durante la infancia no es un proceso lineal en el que éstas emergen de manera progresiva sin vaivenes ni retrocesos, sino que de acuerdo con numerosos estudios empiricos (Kubn, 1995, 2002; Siegler, 1996, 2002) el proceso mediante el cual las estrategias cognitivas emergen y se van consolidando es muy irregular. Consistiría en una secuencia de progresiones y regresiones difícil de captar mediante metodologías estáticas como los diseños clásicos transversales y longitudinales. La perspectiva microgenética aporta una mirada dinámica al proceso de desarrollo y cambio cognitivo. Esta perspectiva utiliza una metodología de recogida de datos de manera intensiva y continuada que permite examinar el cambio mientras éste tiene lugar con el fin de proponer los mecanismos subyacentes al mismo. En el presente trabajo se discuten dos estudios de caso microgenéticos relativos al cambio en las estrategias de adquisición de conocimiento.

Palabras clave: Cambio estratégico, adquisición de conocimiento, competencia metacognitiva, competencia metaestratégica, control de variables, argumentación, microgénesis.

\section{Strategy change in knowledge acquisition: The microgenetic methodology}

\begin{abstract}
The development of cognitive strategies during childhood is not a process in which strategies emerge progressively and linearly. Rather it is formed by a series of temporal patterns of progressions and regressions that make this development irregular (Kubn, 1995, 2002; Siegler, 1996, 2002). This sequence of progressions and regressions is difficult to capture by means of classic cross sectional and longitudinal designs. Microgenetic methodology provides a dynamic view of the developmental process and cognitive change. This methodological perspective uses an intensive and continuous data gathering process that allows change to be examined while it is taking place. The ultimate goal of this methodology is to make proposals of the underlying mechanisms of cognitive change. The present study discusses two microgenetic case studies that analyse change in knowledge acquisition strategies.
\end{abstract}

Keywords: Strategic change, knowledge acquisition, metacognitive competence, metastrategic competence, control of variables, argumentation, microgenesis.

Agradecimientos: El presente trabajo ha sido posible gracias a la financiación de los proyectos SEJ2006-15639-C02-02/ y PR2008-0155 y la Beca FI a la segunda autora del Departamento de Educación y Universidades de la Generalitat de Catalunya y del fondo Social Europeo de la Generalitat de Catalunya. También queremos agradecer de manera especial a los alumnos de la Escuela Piaget de Barcelona así como del Instituto Gabriel Ferrater de Altafulla su participación en la investigación cuyos datos se presentan en este artículo. Sin su participación esta investigación no habría sido posible. Correspondencia con las autoras: Universidad de Barcelona. Facultad de Psicología. Passeig de la Vall d'Hebron, 171. 08035 Barcelona. E-mails: mgarciamila@ub.edu - sandra.gilabert@ub.edu - estellarojo@hotmail.com 
A pesar de que la comprensión de los mecanismos del cambio cognitivo es uno de los aspectos centrales en la psicología del desarrollo, los diseños clásicos transversales y longitudinales para observar dicho cambio sólo permiten captar estados estáticos en distintos momentos y/o en distintas edades del niño a partir de los cuales se intenta describir el proceso e inferir el mecanismo. Paradójicamente, estos diseños contrastan con la metodología utilizada por dos de los fundadores de la psicología del desarrollo, Piaget y Vygostky, los cuales basaban sus investigaciones en la toma de medidas intensivas y repetidas en el momento del cambio. Y aunque el concepto "microgénesis" fuese introducido por Werner (1956), vemos que tanto Piaget (1951) como Vygotsky (1930/1978) ya lo utilizaban para explicar los procesos de desarrollo. En los años 80, la metodología microgenética fue rescatada del olvido por una minoría de psicólogos evolutivos (Kuhn y Ho, 1980; Kuhn y Phelps, 1982) pero no ha sido hasta las últimas décadas que se ha ido imponiendo entre los trabajos que se plantean el análisis de los procesos de cambio cognitivo, así como de la variabilidad que caracteriza dichos procesos. Actualmente, tal como queda patente en el reciente número monográfico en la revista Infant and Child Development (2007) sobre la metodología microgenética o publicaciones específicas sobre la microgénesis (Granott y Parziale, 2002; Siegler, 2002), se observa una reorientación metodológica de la psicología del desarrollo.

Un análisis de los trabajos publicados en los últimos años muestra este viraje desde los estudios tradicionales más cuantitativos cuyo interés se centra en los patrones y tendencias grupales hacia estudios basados en el individuo, estudios de casos que parten de perspectivas más centradas en la variabilidad que en la regularidad como la teoría de los sistemas dinámicos o la teoría microgenética. El presente artículo defiende la idea de que la metodología microgenética aporta información dinámica debido a la naturaleza intensiva de los datos que obtiene. Esta característica permite informar de manera detallada sobre lo que ocurre en momentos de transición en lugar de inferir la información entre los estados inicial y final de la transición (Cheshire, Muldoon, Francis, Lewis y Ball, 2007).

Tal como recogen Flynn, Pine y Lewis (2007), la metodología microgenética requiere el cumplimiento de tres requisitos: (1) Las observaciones deben "cercar estrechamente" el periodo de cambio de la competencia objeto de análisis. Para ello es necesario que el investigador tenga una previsión del periodo temporal en el que el desarrollo de la competencia tiene lugar, así como de las características de la conducta objeto de observación para poder interpretar cualquier cambio en relación a dicha conducta. (2) Se deben generar datos minuciosos en una recogida de datos repetida y intensiva en la que densidad de las observaciones es alta respecto la velocidad del cambio. Se proporciona al participante una tarea durante varias sesiones cuya solución despliega las estrategias objeto de análisis. Se toman medidas repetidamente para registrar estabilidad, continuidad, y cambios en la conducta observada. Finalmente, (3) las observaciones registradas del cambio en la conducta deben someterse a un análisis intensivo con el fin de captar el proceso subyacente que lo produce. El periodo de tiempo, así como el número de sesiones puede variar mucho de un diseño a otro. Pueden ser diseños de una sola sesión de 30 minutos de duración (Bjorklund, Coyle y Gaultney, 1992) o bien de múltiples sesiones, con varios encuentros semanales con una duración de varios meses (Kuhn, Garcia-Mila, Zohar y Andersen, 1995; Kuhn, Schauble y Garcia-Mila, 1992; Siegler y Jenkins, 1989).

La metodología microgenética presenta varios puntos fuertes. Por un lado permite analizar el cambio en directo, mientras se está produciendo. Proporciona datos sobre el recorrido, la velocidad, la amplitud, la variabilidad y lo que origina el cambio. Además permite observar la variabilidad intraindividual y por último, el método microgenético tiene gran flexibilidad teórica. No restringe la perspectiva teórica desde la que se aborda el análisis (Miller y Coyle, 1999). 
En contraste con las ventajas, existen dos aspectos generalmente objeto de crítica. El primero es la artificialidad provocada por la repetición de los encuentros con la tarea. Este reencuentro repetido con la tarea podría provocar un cambio que en situaciones naturales no se daría. Aquellos trabajos que proponen tareas con una periodicidad parecida a la que se presentaría en situaciones naturales serían las menos susceptibles a la crítica. La otra crítica hace referencia a si los procesos de cambio son los mismos a corto que a largo plazo. Es decir, si la microgénesis coincide con la ontogénesis (Grannot y Parziale, 2002). Algunos trabajos han constatado la coincidencia entre la ontogénesis y la microgénesis al proponer un doble diseño microgenético en un diseño transversal en el que se trabaja con dos edades distintas (Kuhn et al., 1995).

Un ámbito del desarrollo cognitivo en el que la metodología microgenética ha resultado particularmente interesante es el estudio de las estrategias de adquisición de conocimiento. Entendemos las estrategias de adquisición de conocimiento como el conjunto de estrategias que permiten un cambio en la comprensión del mundo (Kuhn, 2002; Schoenfeld, 1999). Esto nos conduce al análisis de las teorías que construye el niño como vía para comprender mejor aquello que le rodea. Estas teorías van revisándose a medida que el niño interactúa con su entorno y se encuentra con evidencia que provoca un cambio teórico (Wellman y Gelman, 1992). Pero ¿cómo se revisa el conocimiento? Los diseños microgenéticos permiten observar la evolución de la estructura de conocimiento al coordinar nueva información con conocimientos previos y por el otro permite observar el cambio estratégico en la construcción de dicho conocimiento. Este segundo tipo de cambio es el que se aborda en el presente trabajo.

Las estrategias de adquisición de conocimiento se ponen de manifiesto en contextos de investigación empírica. De acuerdo con el modelo de Kuhn (2002; Kuhn et al., 1995), defendemos que la adquisición de conocimiento en contextos de aprendizaje por descubrimiento se realizaría mediante estrategias agrupadas en 5 fases: Investigación, Análisis, Inferencia, Argumentación y Valor Epistemológico. Cada una de estas fases implica el uso de un conjunto diverso de estrategias, cuyo uso va evolucionando con la práctica y el tiempo. En la fase de Investigación, el niño reconoce e identifica la existencia de una o varias preguntas cuya respuesta está en los datos a los que tiene acceso. En esta fase, el niño debe acceder a los datos, reconocer su relevancia para poner en cuestión sus teorías y formular preguntas sobre estos. La fase de Análisis consiste en hacer accesibles los datos y representarlos como tales (como algo opuesto a la teoría) para establecer comparaciones y patrones. Mientras que la fase de Inferencia se refiere a la aplicación de dichos patrones a la revisión de las teorías previas. Realizar afirmaciones justificadas, refutar afirmaciones no justificadas y reconocer afirmaciones indeterminadas. La cuarta fase, Argumentación, consiste en utilizar las inferencias de la fase anterior en un marco de debate de múltiples alternativas, ya sea intraindividualmente como entre individuos (Garcia-Mila, Andersen y Rojo, 2009).

Finalmente, la quinta fase tratada sólo de manera introductoria en el trabajo de Kuhn (2002), pero plenamente incorporada en el artículo de 2008 (Kuhn, Iordanou, Pease y Wirkala, 2008) corresponde al Valor Epistemológico. Esta fase consiste en las creencias epistemológicas sobre cómo se general el conocimiento científico (Hofer y Pintrich, 1997). Estas creencias epistemológicas subyacen en la aplicación de las estrategias descritas en las fases anteriores. La necesidad de aplicar unas estrategias u otras dependerá de la creencia del niño o el adolescente sobre cómo se construye el conocimiento. Por limitaciones de extensión, esta fase no se abordará en el presente trabajo.

El objetivo del presente trabajo es describir el potencial de la metodología microgenética para capturar el proceso de desarrollo de las estrategias de adquisición de conocimiento descritas, así como proponer el mecanismo de cambio que daría lugar a dicho desarrollo. Dividiremos esta exposición en dos subapartados, correspondientes respectivamente a dos proyectos microgenéticos. El primer proyecto se refiere a datos sobre el desarrollo de las estrategias correspondientes a las fases de Investigación, Análisis e Inferen- 
cia. En el segundo proyecto ilustraremos el desarrollo de las estrategias implicadas en la fase de Argumentación.

\section{Estudio de caso 1. Investigación, Análisis e Inferencia}

En este primer estudio presentamos datos de un participante seleccionado de una muestra de 34 alumnos de $6^{\circ}$ curso de enseñanza primaria que participaron en la investigación (Garcia-Mila et al., 2009). El objetivo del estudio consistió en analizar cómo los preadolescentes averiguaban el funcionamiento de un sistema multicausal sobre el que inicialmente sostenían una serie de teorías (crecimiento de las plantas). Se trabajó con los preadolescentes en sesiones individualizadas durante varias semanas en las que en cada sesión se les preguntaba sobre su conocimiento previo acerca de las variables del sistema a investigar y se les pedía que diseñaran experimentos que más tarde debían analizar e interpretar. El diseño microgenético nos permitió observar cómo el conocimiento se iba modificando en relación al cambio en las estrategias de recogida y manipulación de los datos (investigación empírica) y en las estrategias de análisis e inferenciales (Garcia-Mila et al., 2009). La tarea se presentó a los participantes como el siguiente problema científico.

Nuestro gobierno quiere probar una semilla (Brassica) que ya ha sido probada previamente en otro país con resultados excelentes para alimentar el ganado. Además el Gobierno tiene otra semilla (Rosetta), muy similar a Brassica, que podría ser tan eficiente como ella, y que además es mucho más barata. Tu tarea consiste en determinar las condiciones en las que la planta producirá una mejor cosecha. Deberás decidir cuál de las dos semillas da un mejor resultado, así como decidir si es mejor utilizar un fertilizante químico o ecológico y si es mejor la luz natural del sol o la luz artificial de un invernadero

Se proporcionó a los participantes el material necesario para llevar a cabo la experimentación. Tiestos, tierra, fertilizante de dos tipos (químico/Q vs. ecológico/E), los dos tipos de semillas (Brassica/B vs. Rosette/R), y un entorno de laboratorio con dos zonas delimitadas, una al lado de la ventana y la otra debajo de una luz fluorescente (luz natu$\mathrm{ral} / \mathrm{N}$ vs. luz artificial/A). También se les proporcionaron etiquetas y un bloc de notas para apuntar todo aquello que creyeran oportuno. Los participantes trabajaron durante siete sesiones. En las cuatro primeras diseñaron los experimentos (cuatro plantas en las sesión 1, cuatro en la sesión 2 y dos en la sesión $4^{1}$ ), y en todas las sesiones excepto en la primera, pudieron observar, analizar y hacer inferencias sobre los resultados de sus experimentos.

Un valor importante de esta investigación se encuentra en el hecho de que se pidió a los participantes realizar el ciclo completo de la investigación para la adquisición de conocimiento: diseño experimental y análisis de los datos para hacer inferencias y llegar a conclusiones de manera repetida de forma que el feedback de los resultados de los experimentos podía actuar de ayuda a la reflexión sobre la eficacia estratégica y su mejora. Esta característica permite analizar las estrategias de las distintas fases desde el punto de vista de su interacción mutua. Para la resolución de la tarea el participante debe plantearse las preguntas objeto de la investigación. Una vez formuladas la preguntas, éste debe de recoger los datos, es decir diseñar los experimentos que le permitirán responder a dichas preguntas. Finalmente, debe analizar los datos obtenidos y hacer inferencias para llegar conclusiones para revisar sus teoría.

Para una recogida de datos rigurosa se deben de aplicar dos estrategias: la combinación factorial de variables y el control de variables, mientras que en la fase de interpretación de los datos, implica generar inferencias válidas. Tanto el control de variables (Chen y Klahr, 1999; Tschirgi, 1980) como la combinación factorial de variables (Inhelder y Piaget, 1958) han sido estrategias ampliamente investigadas en la literatura del desarrollo cognitivo mediante diseños transversales y el consenso apunta a la preadolescencia como el periodo durante el cual emergen y se van consolidando (ver Zimmerman, 2000, 2005 para una revisión). Ambas estrategias son esenciales en el sentido 
de que no sólo permiten explorar todo el espacio problema (Klahr, 2000), sino que permiten hacer inferencias válidas. Según la estrategia de control de variables, en el diseño de dos experimentos controlados, se deben mantener idénticas todas las variables del sistema causal excepto aquella cuyo efecto se pretende determinar. Es lo que Tschirgi (1980) denomina la estrategia VOTAT (vary-one-thing-at-a-time). Los experimentos que no se basan en el control de variables no pueden derivar en inferencias válidas ya que los resultados que generan no son concluyentes respecto al efecto de las variables. Mediante análisis de caso (Jorge ${ }^{2}$ ) observaremos cuál es el proceso de apropiación de ambas estrategias empíricas, y las inferencias que se aplican para el análisis de los datos que éstas generan.

En la primera sesión se le pregunta a Jorge por sus teorías previas, y defiende con contundencia su teoría de lo natural mejor que lo artificial. Defiende que la luz natural y el abono ecológico serán mejores para la planta que el abono químico y la luz artificial, respectivamente. Más adelante veremos cómo esta teoría llevará a una aplicación sesgada de las estrategias. Por otro lado defiende que la semilla Brassica crecerá mejor que a Rosette. La pregunta de partida es fundamental en el proceso experimental, ya que las estrategias seleccionadas están ligadas al objetivo del experimento diseñado. Uno de los objetivos que más sesga el diseño de experimentos es lo que Tschigi (1980) denomina "generar el mejor resultado". Así es como procede Jorge. En el primer experimento, propone plantar Brassica con luz natural y abono ecológico (BNE). Para Jorge, ésta es la combinación que supuestamente dará mejor resultado. Es en el diseño del segundo experimento cuando ya podemos observar si Jorge contempla la necesidad de ir combinando los factores sistemáticamente para completar todo el espacio problema y a su vez diseñar un experimento basado en la estrategia de control de variables. Cuando se le pregunta qué quiere poner en la segunda maceta, responde:

Jorge- ... pues en ésta pongo la Rosette. Y asípodré comprobar la diferencia entre la Brassica y la otra Rosette.. Las pondré en las mismas condiciones, y así veré si en una con las mismas condiciones (BNE) crece más que la otra (RNE) (...) Pondré Rosette, y también con luz natural y abono ecológico (RNE) (...) Para averiguar, en las mismas condiciones, cuál crece más de las dos semillas

Exp.¿Quécrees que pasará?

Jorge-. No lo sé, por que nunca he oído hablar de estas semillas

Aunque al inicio de la investigación, cuando se le pregunta a Jorge por las teorías previas manifiesta que la semilla Brassica es mejor que la Rosette, el último comentario nos hace pensar que ésta no es una teoría tan arraigada como la de "lo natural" mejor que "lo artificial". Esta neutralidad teórica le permite aplicar aparentemente sin problemas la estrategias de control de variables, pues en el primer par de experimentos ha controlado todos los factores excepto el de la semilla (BNE vs. RNE). Para darle la oportunidad de explicitar la estrategia, se le pregunta sobre lo que pretende averiguar con este experimento, y se observa que efectivamente hay una intencionalidad de controlar variables, una conciencia de necesidad experimental para poder determinar el efecto de las variables, en este caso, de la semilla. Esta falta de teoría previa que podría sesgar el diseño experimental cambia radicalmente en el diseño del siguiente par de experimentos, cuando entra en juego su teoría de "lo natural mejor que lo artificial". Veamos cómo Jorge cambia de estrategia al diseñar el siguiente par de experimentos, en el que el objetivo pasa a ser la comprobación de su teoría sobre lo natural mejor. Cuando se les pregunta por las dos macetas siguientes, responde: La Brassica, pero que tenga luz artificial y abono quimico (BAQ), y la respuesta al preguntarle sobre lo que pretende averiguar contesta: para ver si la de luz natural y abono natural (ecológico) crecerá más que la artificial (BAQ).

En la cuarta maceta, Jorge pone los mismos que en la tercera cambiando la semilla (RAQ). Es decir que vuelve a tener un par de experimentos con la semilla controlada. No obstante para poner a prueba su teoría de "lo natural mejor" observamos que cambia 
dos factores a la vez, contrapone los dos niveles naturales de la variable (luz natural y abono ecológico) con los dos niveles artificiales (luz artificial y abono químico), violando el principio del control de variables. Jorge es bastante sistemático al diseñar experimentos, parece que tiene claro el objetivo de su investigación. Mediante el primer par pone a prueba la teoría de la semilla, y mediante el segundo par la teoría de lo natural. No obstante vemos que lo que parecía una estrategia de control de variables relativamente sólida, se pone en peligro por causa de su teoría fuertemente arraigada sobre lo natural mejor que lo artificial. Cuando se le pide que explique qué ha hecho, responde: Poner las dos Brassicas y las dos Rosettes, y todas en las mismas condiciones para ver que las naturales crecerán más que las no naturales.

En la segunda sesión, tiene delante todas las plantas sembradas en la sesión anterior y concluye que su teoría de lo natural mejor no ha funcionado y para proteger su teoría, la matiza planteando que la mezcla entre lo natural y artificial será mejor que todo natural. Para probar su nueva teoría, cambia de estrategia: diseña las dos posibles mezclas de natural con artificial, ignorado el control de variables completamente mostrando una regresión importante respecto al control de variables. Pone una maceta con luz natural con abono químico y otra con luz artificial con abono ecológico en la Brassica primero y en la Rosette después (BNQ, BAE, RNQ, RAE).

Tal como hemos mencionado anteriormente, el valor añadido del presente diseño es que no sólo permite observar las estrategias del participante a los largo de las sesiones, sino que permite relacionar las estrategias implicadas en las distintas fases (investigación, análisis e inferencia). Veamos pues las estrategias inferenciales, y podremos constatar una vuelta al uso del control de variables implícito para hacer inferencias válidas. Analicemos las observaciones, análisis e inferencias de Jorge en la sesión $5^{a}$, sesión en la que tiene toda la base de datos delante?

Jorge- en 14 dias, ésta (BNE) ha crecido $10 \mathrm{~cm}$ (..) Y la que más, bueno no es la que más ha crecido, pero la que mejor color tiene y parece más gordo el tronco y las hojas es mejor, todo mejor es la otra BAE del día 15, y ba crecido 4 cm y han salido dos plantas. Ya está!

Exp.-¿Qué has averiguado con estos experimentos?

Jorge- Pues que a la B, al ser más larga, le va bien la luz natural y el abono ecológico (BNE), pero para que salga con un buen color, unas buenas hojas y un tronco parece bastante resistente, necesita la luz artificial y el abono ecológico. O sea, la luz a estas dos les ha afectado diferente: La luz artificial a la Brassica le hace coger más color y crece mejor.

Exp.-¿Cómo sabes que es la luz que produce este efecto?

Jorge-Comparando esta planta (BNE) que ba crecido mucho y tiene un tronco muy débil y en cambio, ésta (BAE) que parece con un poco más gordo y tiene unas hojas con mucho más color y más vivas). (...) Que a la Brassica lo que mejor le va es esto, la combinación de la luz artificial y el abono ecológico (BAE de la $2^{\mathrm{a}}$ sesión).

Vemos en el fragmento anterior que Jorge hace una inferencia válida (basada en el control de variables) sobre la luz que sirve para refutar su teoría previa de natural mejor que artificial. Por tanto, la estrategia de control de variables o al menos la necesidad de basar las inferencias en estrategias del control de variables vuelve a aparecer, al menos de manera implícita. Sin embargo, cuando se le pregunta que interprete el resultado, vuelve a su nueva teoría (combinación de natural con artificial es lo que le va mejor) que funcionaría como protección de su teoría inicial fuertemente arraigada de lo natural mejor y con gran resistencia a ser abandonada. En la sesión 6 vuelve a observar y dice:

Jorge- La Brassica del día 15, la que hay más antigua, de abono químico (BNQ) no ba crecido y la última del día 22 de BNE ha crecido. Habia dos, una ha crecido $2,5 \mathrm{~cm}$ y la otra $1 \mathrm{~cm}$. La RAQ nada, la RAQ nada y la última, la BAE es la que mejor color tiene y es la que más ha crecido, ha crecido $6 \mathrm{~cm}$

Exp.-¿Qué has averiguado con estos experimentos?

Jorge- Que la Rosette parece ser que ninguna condición le ha ido bien, ninguna de las condiciones en las que la hemos sometido iguales de temperatura, y que a la Brassica, sí! También be mirado, una con luz natural, el mismo abono y una de luz artificial. La del luz natural crece más rápido pero con el tronco más endeble y con menos hojas (comparación aparentemente basada en el control de variables) en cambio las de luz artificial crecen mucho mejor (inferencia generalizada). 
En la parte final del fragmento anterior observamos una regresión de una inferencia válida a un inferencia general, a pesar de que tiene posibilidad hacer inferencias válidas para cada uno de los factores ya que su sistematicidad en la recogida de datos le ha permitido completar todas las combinaciones posibles. Jorge se limita a hacer inferencias sobre sus teorías centrándose sólo en los datos que le ayudan a confirmarlas. Cuando se le pide que interprete el efecto, responde: porque con las de abono químico no ha pasado (el plural de "las" vuelve a mostrar una inferencia general).

La variabilidad en la aplicación de estrategias inferenciales mostrada por Jorge iría desde la ausencia de inferencias, pasando por inferencias en las que los datos son ignorados (hay factores cuyo efecto ha ignorado totalmente), o bien en las que éstas utilizan el ejemplo como justificación de la teoría. Entre las estrategias más avanzadas, estarían las inferencias justificadas mediante datos concluyentes confirmatorios, y finalmente inferencias que identifican datos refutativos y que los utilizan para refutar sus teorías previas. Es sorprendente como los datos muestran una coexistencia entre todos los tipos de inferencias, coexistencia que incluye desde las más avanzadas a las más primitivas tal como lo muestran los ejemplos anteriores.

Dado que el estudio del que hemos extraído los ejemplos para ilustrar la variabilidad intraindividual era de investigación autodirigida y se realizaba en sesiones individuales, el diseño no permitió observar a los participantes en un contexto de debate en el que pudieran poner de manifiesto su capacidad argumentativa. Para ilustrar la variabilidad en la fase de Argumentación presentamos datos de una segundo estudio microgenético.

\section{Estudio de caso 2. Argumentación}

En el segundo estudio contextualizamos una situación de debate sobre el cambio climático con el fin de poner de manifiesto el desarrollo de las estrategias argumentativas. De manera similar al estudio anterior, tomamos un estudio de caso extraído de una muestra de 60 alumnos de $1^{\circ}$ de la ESO (12-13 años) (Felton, Garcia-Mila y Gilabert, 2009). Se presentaron a los alumnos tres dilemas sobre el cambio climático. Los tres dilemas se plantearon en tres clases de ciencias en sesiones consecutivas. Los alumnos trabajaron en díadas y sus discusiones fueros grabadas en audio. Los dilemas estaban basados en conocimiento tratado en clase sobre las causas de cambio climático y sobre conceptos de energías renovables y no renovables ${ }^{4}$.

Nos basamos en los trabajos de Walton (1989) y Felton (2004) para establecer que para una buena argumentación es necesario que el individuo tenga claros tres objetivos: (1) identificar las premisas para justificar una afirmación determinada, (2) identificar las afirmaciones no justificadas y (3) refutar o neutralizar los retos a los propios argumentos (Walton, 1989). Para construir un argumento de manera competitiva en el contexto de un debate es necesario asumir los objetivos anteriores y aplicar estrategias efectivas para lograrlos (Felton, 2004; Felton y Kuhn, 2001). Cuando alguien quiere debilitar la afirmación del contrario, necesita pensar sobre los puntos débiles para reforzar las propias afirmaciones. Por tanto, pera estudiar el desarrollo de las estrategias argumentativas, el análisis se debe centrar en el progreso desde formas argumentativas simples (i.e. No estoy de acuerdo) cuyo objetivo es exponer el punto de vista propio sin tener en cuenta la posición del contrario al uso de contraargumentos y refutaciones, en las que el objetivo pasa a ser tomar conciencia de la debilidad del planteamiento del adversario y reforzar la propia posición. Felton y Kuhn (2001) agrupan estas estrategias argumentativas en dos grandes grupos:

1) Exposición de la posición propia: Articulación y clarificación de la propia posición en el debate mediante estrategias discursivas del tipo clarificar (una demanda del partner para aclarar posiciones) o añadir (hacer una elaboración de la intervención previa del partner sin aportar información nueva. 
2) Estrategias para retar al adversario: Apuntar al discurso del adversario para identificar las debilidades que éste presenta. Estas estrategias empezarían como simples desacuerdos (plantear el desacuerdo con la intervención previa del adversario sin ninguna justificación), y contraargumentos, que pueden consistir en el desacuerdo con la afirmación previa del partner acompañada de un argumento alternativo, o bien un desacuerdo con la afirmación previa del partner acompañada de una crítica a dicha afirmación.

El progreso no tendría lugar de forma lineal y progresiva, sino que igual que en las estrategias de investigación del apartado anterior, avanzaría mediante series de progresiones y regresiones. Este progreso es el que ilustramos mediante el siguiente extracto de estudio caso microgenético. La díada seleccionada para el análisis está formada por Marc y Juan, y dentro de ésta, centraremos la mirada únicamente en Marc, uno de los partners de la díada. En la primera sesión, Marc, ante el dilema sobre si instalar un central térmica de combustión fósil o bien una central nuclear, escoge la primera. Y aunque la justificación de sus argumentos es relativamente sencilla, del tipo:

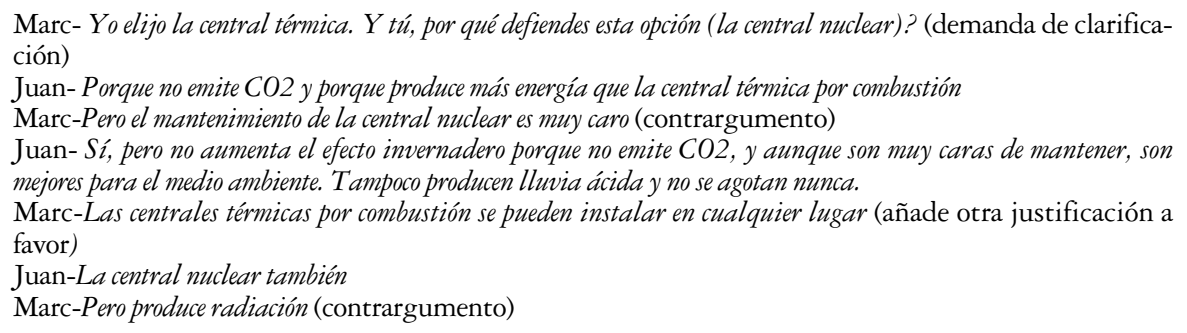

Observamos en este fragmento de la discusión, prototípico del resto del debate, la presencia de argumentos muy sencillos pero justificados y apoyados por contraargumentos, también sencillos pero que dan lugar a respuestas más elaboradas de Juan. Marc es capaz de empezar planteando una demanda de clarificación, responde con un contrargumento, sigue con una justificación para reforzar su propia posición y acaba con otro contrargumento. La presencia de contraargumentos es un paso previo a dirigir el discurso al partner, retando sus argumentos para reforzar las propias posiciones. Observamos por tanto en este fragmento, un potencial de avance de acuerdo con el planteamiento de Walton (1989). Hemos observado en la primera sesión que el debate no incluye las formas más simples de argumentación como las afirmaciones simples y negaciones (desacuerdos simples). Vemos que pasa en la segunda sesión, en la que discuten sobre la instalación de un parque eólico:

Juan-Yo be escogido la opción en contra del parque eólico

Marc-Yo a favor (establece su posición en el debate)

Juan-¿Por qué la has escogido?

Marc- ¿Y tú? (demanda de aclaración)

Juan- Yo la he escogido porque pienso que construir y mantener un parque eólico es muy caro, provoca impacto visual, mueren pájaros y hace ruido

Marc-Si no se pone un parque eólico, qué harás para obtener energía? (demanda de aclaración)

Juan- Pues pondría placas solares en los tejados de cada casa, placas solares resistentes al agua y a todo. Y tú, qué propones?

Marc-No sé... pero con la energía solar no se puede abastecer a toda la población (no ha justificado su posición, pero las demandas anteriores de aclaración le han proporcionado argumentos para responder con contraargumentos)

Juan-Pero podríamos poner energía solar, hidráulica y biocombustibles

Marc- ¿Biocombustibles? (demanda de aclaración)

Juan-No, los biocombustibles no! Solar y hidráulica, la hidráulica es la del agua

Marc-Pues no! Yo quiero la eólica (afirmación simple)

Juan- Pues no! la solar o la bidráulica.

Marc- $¿ Y$ el día que no haga sol? (demanda de aclaración)

Juan-Ponemos una central hidráulica y placas solares en la casas del Baix Gaià, cuando no haga sol utilizamos la energía que se genera en la central hidráulica y cuando haga sol... 
Marc- ¿Y si no hay agua? (demanda de aclaración). (...) Yo aún sigo pensando que la instalación de un parque eólico es buena idea (afirmación simple)

Juan-Pues yo opino que no, que no se tiene que poner un parque eólico! Los parques eólicos provocan impacto visual, podría afectar el ecosistema, podrían desaparecer especies de pájaros, es muy caro de mantener y hacen mucho ruido. Te ha quedado claro? Te lo repito otra vez (...)

Marc-Vale, pues si no instalamos un parque eólico, qué hacemos? (...) Yo creo que es mejor la solar (afirmación simple)

Juan-Pues yo escojo la bidráulica

Marc-Yo solar (afirmación simple)

El diálogo sigue con el mismo patrón repetitivo sin información nueva añadida. Observamos una regresión clara respecto al fragmento de la $1^{a}$ sesión. Hemos pasado de alguna afirmación simple y demanda de aclaración matizada con contraargumentos a un diálogo en el que predominan las afirmaciones y negaciones simples, combinadas con demandas de aclaración.

En la tercera sesión Juan y Marc debaten sobre biocombustibles. Marc se posiciona a favor de la instalación de una fábrica de biocombustibles en el pueblo, mientras que Juan defiende la opción en contra de la fábrica vemos indicadores de avance del discurso.

Juan- Por qué has escogido la opción fábrica de biocombustibles?

Marc- Porque los biocombustibles no emiten CO2, por tanto no tiene incidencia en el efecto invernadero (afirmación justificada)

Juan-Yo estoy en contra porque el combustible que proviene de la biomasa es muy caro y se necesitan muchas tierras para poder cultivar la biomasa. También se utilizan fertilizantes que pueden dañar el medio ambiente.

Marc- Pero no tanto como una central nuclear! (contrargumento)

Juan- Pero los biocombustibles también son dañinos para el efecto invernadero

Marc-Pero muy poco... (contrargumento)

Juan- Se tiene que cortar árboles y plantas para cultivar la biomasa. Los países pobres se volverán aún más pobres. ¿A ver, qué tiene que decirme a esto?

Marc. Y si no optamos por los combustibles, que fuente de energía propondrías? (demanda de aclaración)

Juan-Yo opto por la hidráulica y la solar

Marc-Pero con esta fuente de energía no se generaría la energía eléctrica ni el combustible para el consumo actual (contrargumento)

Juan-Yo propondría construir coches que funcionasen con electricidad, y así no necesitarían combustible, simplemente necesitarían agua y sol

Marc-Esta costaría mucho dinero (contrargumento). ¿Quién lo compraría? (demanda de aclaración) (..) A mí me gusta la solar (afirmación simple), pero sigo pensando que los biocombustibles son una buena opción (afirmación simple)

Juan-¿Por qué?

Marc-Porqué no emiten CO2, no aumenta el efecto invernadero y la biomasa que se utiliza es renovable y no genera lluvia ácida (repetición de afirmación justificada)

La discusión entra en una fase de repetición de argumentos tanto científicos como económicos para llegar al consenso que propone placas solares a pesar de que son más caras. Los fragmentos de los debates presentados muestran la desigual aplicación de las estrategias discursivas. Un parámetro muy ilustrativo y complementario a las justificaciones son las afirmaciones i/o negaciones simples del tipo: pues no! Yo quiero la eólica (ver diálogo). Hemos visto que no estaban presentes en el primer diálogo (0), aumentaban en el segundo (7) para volver a bajar en el tercero (3), mientras que las afirmaciones justificadas varían de manera inversa. En la primera sesión observamos 13 afirmaciones justificadas sobre 18 turnos. En la segunda sesión observamos sólo sobre 1 sobre 21 turnos y en la tercera sesión contabilizamos 9 sobre 24 turnos o intervenciones (ver Felton et al., 2009 para el análisis cuantitativo).

También las intervenciones que piden clarificación, a menudo como producto de la necesidad de obtener argumentos para seguir la discusión aparecen de manera desigual. Finalmente, otro indicador de desarrollo de estrategias argumentativas es el uso de contraargumentos, los cuales también hemos visto cómo aparecen en el primer diálogo, bajan en el segundo drásticamente, para volver a aparecer en el tercero. 


\section{Discusión}

Los dos estudios de caso nos han permitido observar el irregular proceso de apropiación de estrategias de adquisición de conocimiento, tanto en lo que se refiere a las estrategias de investigación empírica (diseño experimental, análisis e inferencia) en un contexto de investigación autodirigida como en lo que se refiere a las estrategias argumentativas en un contexto de debate. Hemos podido ver el amplio espectro de diferentes estrategias que se aplican de manera muy variable a una misma tarea a lo largo del tiempo, observando una sucesión de progresiones y regresiones temporales. Hemos podido observar también el grado de dependencia que estas estrategias tienen de las teorías subyacentes, de cuánto debe desmarcarse el individuo respecto a sus teorías, y a su vez, de cuán consciente debe de ser de la necesidad de coordinar teoría y datos. Estrategias nuevas y cada vez más eficaces van surgiendo en los distintos encuentros con la tarea, a costa del abandono de las estrategias viejas, de forma que el cambio cognitivo se observa como una cambio gradual en la distribución de uso de las estrategias, con las más eficaces tomando terreno y las menos eficaces utilizándose progresivamente menos (Kuhn, 2002; Kuhn et al., 1995; Siegler, 1995, 2006).

La presencia de regresiones en el contexto de un cambio de estrategia progresivo debe interpretarse como algo positivo. Estamos de acuerdo con Siegler (1995) cuando plantea que recurrir a estrategias menos avanzadas después de construir y aplicar estrategias nuevas, más avanzadas, podría interpretarse como una "regresión positiva". El surgimiento de una estrategia no determina su aplicación consistente, sino que ésta debe competir con estrategias del repertorio menos adecuadas o eficaces. Esta competición sería la responsable de las regresiones puntuales hacia estrategias viejas. Los niños necesitarían reforzar o automatizar los componentes que convergen en las estrategias más avanzadas, o bien, tal como apuntan Miller y Cole (1999), deben asegurarse de que las estrategias menos avanzadas ya no son tan útiles. El cambio cognitivo surgiría como resultado del abandono de las estrategias viejas, menos eficaces que pasarían a ser consideradas por el sistema cognitivo como poco útiles y por tanto, obsoletas. La comprensión de metanivel entendida como la comprensión de porqué una estrategia es más efectiva que sus estrategias alternativas es crucial para explicar dicho abandono.

Miller y Coyle (1999) proponen tres cambios particularmente interesantes para comprender el desarrollo estratégico en general: (1) la emergencia de una nueva competencia o nueva comprensión conceptual; (2) la producción eventual de una competencia pre-existente durante la solución de un problema, y (3) la progresiva presencia a lo largo de los distintas sesiones de esta nueva o pre-existente competencia. De manera implícita en el planteamiento de estas autoras encontramos la idea subyacente de la importancia de comprender la selección de estrategias, además de su ejecución. Uno de los aspectos fundamentales en el desarrollo sería no tanto el dominio de nuevas y más sofisticadas estrategias, sino el abandono de las ya adquiridas, más familiares, cómodas de aplicar y seductoras. El cambio cognitivo lo interpretamos en función de la regulación metacognitiva mediada por el feedback que proporciona el encuentro repetido con la tarea. Este feedback sería fundamental en todo el proceso pues aumentaría la conciencia de cuán correctamente se estaría cumpliendo el objetivo de la tarea mediante las estrategias seleccionadas y su forma de aplicación, reconociendo las ventajas y limitaciones de cada una. Esta conciencia sería la que comportaría la posterior selección de otras estrategias del repertorio o bien la búsqueda de nuevas. Es decir, además de centrarnos en el nivel de competencia en la ejecución, para poder comprender el cambio debemos enfocar al nivel metacompetencial en la selección de una estrategia de todo el abanico disponible. Así pues el desarrollo de las estrategias de adquisición de conocimiento podría explicarse a partir de avances en la competencia estratégica, competencia metaestratégica y competencia metacognitiva (Garcia-Mila y Andersen, 2007; Kuhn, 2002). La competencia estratégica se refiere a la habilidad para aplicar y ejecutar la estrategia correcta. Las 
nuevas estrategias se van reforzando con la práctica, la cual permite que las estrategias menos avanzadas vayan siendo sustituidas por las nuevas durante la microgénesis. $L a$ competencia metaestratégica se referiría a saber cómo, cuándo y por qué de debe aplicar una estrategia y no otra en relación a la representación de la tarea y sus objetivos. En esta competencia se ponen en juego procesos de autorregulación estratégica en el repertorio de cada uno, seleccionado estrategias a partir de su eficacia y abandonando otras por su ineficacia. La competencia metacognitiva se refiere a revisar las teorías a partir de evidencia empírica. A menudo no se distingue la evidencia como algo separado de la teoría, y que puede ser refutada, especialmente cuando la teoría está muy arraigada, es contundente y los datos son ambiguos. En este caso el investigador acostumbra a interpretarla con un fuerte sesgo confirmatorio tal como hemos visto en el caso de Jorge y su teoría de lo natural mejor que lo artificial, para pasar al matiz de combinar artificial y natural.

En la línea de otros trabajos del presente monográfico (van Dijk y van Geert, 2011), entendemos que una de las nuevas direcciones que la metodología microgenética es su combinación con la teoría de los sistemas dinámicos. Tal como plantean Millar y Coyle (1999) ambas metodologías tienen el potencial de enriquecerse mutuamente para comprender el cambio cognitivo. Por un lado, la teoría de los sistemas dinámicos aborda el cambio a lo largo del tiempo en sistema complejos, aportando una mirada a la emergencia de nuevas competencias integrando todas las partes implicadas. Se defiende la idea que una pequeña diferencia inicial puede tener reverberaciones que podrían culminar en diferencias importantes más tarde. También establece que se debe examinar la conducta y las diferencias individuales no sólo en el momento que precede el cambio observado, sino desde mucho antes. Finalmente dado que un cambio pequeño puede acusar cambios en todo el sistema, según la teoría de los sistemas dinámicos, los experimentos microgenéticos deberían ampliar las conductas objeto de análisis. De acuerdo con Thelen (1992), los cambios microgenéticos en la apropiación de estrategias deben ser considerados como un intento de coordinar las competencias del niño, con sus motivaciones y con las demandas de la tarea.

En sentido contrario, la metodología microgenética puede contribuir a la teoría de los sistemas dinámicos. Los resultados de la aplicación de la metodología microgenética permite, tal como hemos visto, una secuencia detallada del cambio, momento a momento y individuo a individuo para captar las diferencias intraindividuales. Los resultados empíricos permiten establecer los principios de cambio cognitivo, regresiones temporales frente a nuevas situaciones y la sensibilidad de los procesos a los objetivos de la tarea tal como los interpreta el individuo, la motivación y la metacognición.

\section{Notas}

${ }^{1}$ Los participantes dedicaron la sesión 3 a observar y a escribir un informe científico de los resultados obtenidos.

${ }^{2}$ Los nombres reales han sido sustituidos por pseudónimos.

${ }^{3}$ En la $5^{\mathrm{a}}$ sesión Jorge tiene los cuatro experimentos de la $1^{\mathrm{a}}$, los cuatro de la $2^{\mathrm{a}}$, y los dos de la $4^{\mathrm{a}}$. Tiene en total una base de datos formada por diez experimentos.

El diseño experimental incluyó como requisito que los miembros de la díada estuvieran en desacuerdo inicial respecto a las dos posiciones planteadas en el dilema.

\section{Referencias}

BJorklund, D. F., COYle, T. R. \& GAultney, J. F. (1992). Developmental differences in the acquisition and maintenance of an organizational strategy: Evidence for the utilization deficiency hypothesis. Journal of Experimental Child Psychology, 54 , 434-438.

CHEN, Z. \& KLAHR, D. (1999). All other things being equal: Acquisition and transfer of the control of variable strategy. Child Development, 70, 1098-1120.

Cheshire, A., Muldoon, K. P., Francis, B., Lewis, C. M. \& Ball, L. J. (2007). Modelling Change: New opportunities in the analysis of microgenetic data. Infant and Child Development, 16, 119-134.

Felton, M. (2004). The development of discourse strategies in adolescent argumentation. Cognitive Development, 19, $35-52$. 


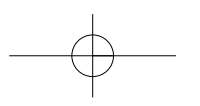

Felton, M., Garcia-Mila, M. \& GILABert, S. (2009). The impact of discourse goals on content learning and argumentation in science. Paper presentado al European Association for Research on Learning and Instruction (EARLI), Agosto, 2009, Amsterdam, The Netherlands.

Felton, M. \& KuHN, D. (2001). The development of argumentive discourse skills. Discourse Processes, 32, 135-153.

FLYNN, E., PINE, K. \& LEWIS, C. (2007). Using the microgenetic method to investigate cognitive development: An Introduction. Infant and Child Development, 16, 1-6.

Garcia-Mila, M., Andersen, C. \& Rojo, N. (2009). Representational practices and scientific inquiry. En C. Anderson, N. Scheuer, M. P. Pérez Echeverría \& E. Teubal (Eds.), Representational Systems and Practices as Learning Tools in Different Fields of Knowledge (pp. 167-187). Rotterdam: Sense Publishers.

Granott, N. \& PARZIAle, J. (Eds.) (2002). Microdevelopment: Transition processes in development and learning. Cambridge, UK: Cambridge University Press.

Hofer, B. \& Pintrich, P. (1997). The development of epistemological theories: Beliefs about knowledge and knowing and their relation to learning. Review of Educational Research, 67, 88-140.

INHELDER, B. \& PIAGET, J. (1958). The growth of logical thinking from childhood to adolescence. Nueva York: Basic Books.

Karmiloff-Smith, A. (1984). Children's problem solving. En M. Lamb, A. L. Brown \& B. Rogoff (Eds.), Advances in developmental psychology (Vol. 3, pp. 39-89). Hillsdale, NJ: Lawrence Erlbaum Associates.

KLAHR, D. (2000). Exploring science: The cognition and development of discovery processes. Cambridge, MA: The MIT Press.

KuHN, D. (1995). Microgenetic study of change: What has it told us? Psychological Science, 6, 133-139.

KuHN, D. (2002). A multi-component system that constructs knowledge: Insights from microgenetic study. En N. Granott \& J. Parziale (Eds.), Microdevelopment: Transition processes in development and learning (pp. 109-131). Cambridge, UK: Cambridge University Press.

KuHN, D. \& Ho, V. (1980). Self-directed activity and cognitive development. Journal of Applied Developmental Psychology, 1, $119-133$.

Kuhn, D., Garcia-Mila, M., Zohar, A. \& Andersen, C. (1995). Strategies of knowledge acquisition. Monograph of the Society for Research in Child Development, 60 (4), Serial No.245.

Kuhn, D., Iordanou, K., Pease, M. \& Wirkala, C. (2008). Beyond control of variables: What needs to develop to achieve skilled scientific thinking? Cognitive Development, 23, 435-451.

KuHN, D. \& PhelPs, E. (1982). The development of problem-solving strategies. En H. Reese (Ed.), Advances in child development and behavior (Vol. 17). Nueva York: Academic Press.

Kuhn, D., Schauble, L. \& Garcia-Mila, M. (1992). Cross-domain development of scientific reasoning. Cognition and Instruction, 9 (4), 285-327.

Miller, P. H. \& COYLE, T. R. (1999). Developmental change: Lessons from microgenesis. En E. K. Scholnick, K. Nelson, S. A. Gelman \& P. H. Miller (Eds.), Conceptual development. Piaget's legacy (pp. 209-241). Mahwah, NJ: Lawrence Erlbaum Associates.

PiAget, J. (1951). Play, dreams and imitation in childhood (2 ed. 1962). Londres: Routledge and Kegan Paul.

SCHOENFELD, A. (1999). Looking toward the 21st century: Challenges of educational theory and practice. Educational Researcher, 28, 4-14.

SIEGLER, R. (1995). How does change occur: A microgenetic study of number conservation. Cognitive Psychology, 25, $225-273$.

SIEGLER, R. (1996). Emerging minds: The process of change in children's thinking. Nueva York: Oxford University Press.

SiEgLER, R. S. (2002). Microgenetic studies of self-explanation. En N. Granott \& J. Parziale (Eds.), Microdevelopment: Transition processes in development and learning (pp. 31-58). Cambridge, UK: Cambridge University Press.

Siegler, R. S. (2006). Microgenetic analyses of learning. En W. Damon \& R. M. Lerner (Series Eds.) \& D. Kuhn \& R. S. Siegler (Vol. Eds.), Handbook of child psychology: Volume 2: Cognition, perception, and language (6 $\left.{ }^{\mathrm{a}} \mathrm{ed}.\right)$ (pp. 464-510). Hoboken, NJ: Wiley.

SIEGLER, R. \& JenKINs, E. (1989). How children discover new strategies. Hillsdale, NJ: Lawrence Erlbaum Associates.

THELEN, E. (1992). Development as a dynamic system. Current Directions in Psychological Science, 1, 189-193.

TsCHIRGI, J. E. (1980). Sensible reasoning: A hypothesis about hypotheses. Child Development, 51, 1-10.

VAN DiJK, M. \& VAN GEERT, P. (2011). Analyzing variability as a dynanic aspect of change. Infancia y Aprendizaje, 34 (2), ......

Vygotsky, L. (1930/1978). Mind in society: The development of higher mental processes. Cambridge: MA: Harvard University Press (Trabajos originales publilcados en 1930,1933 y 1935).

Walton, D. N. (1989). Dialogue theory for critical thinking. Argumentation, 3, 169-184.

WERNER, H. (1956). Microgenesis and aphasia. Journal of A bnormal and Social Psychology, 52, 347-353

Zimmerman, C. (2000). The development of scientific reasoning skills. Developmental Review, 20, 99-149.

ZimMERMAN, C. (2005). The development of scientific reasoning skills: What psychologists contribute to an understanding of elementary science learning. Washington, DC: National Research Council. 